\title{
Densidad de los residuos sólidos de tres instituciones educativas de la ciudad de Chachapoyas, departamento de Amazonas
}

\section{Density of the solid residues of three educational institutions of the city of Chachapoyas, department of Amazonas}

\author{
Enith Mercedes Cabañas Pinedo ${ }^{1 *}$, Maribel Díaz Sánchez ${ }^{1}$, Manuel Oliva1 ${ }^{1}$ (D)
}

\section{RESUMEN}

La presente investigación tuvo como objetivo determinar la densidad de los residuos sólidos generados en las instituciones educativas San Juan de la Libertad, Virgen Asunta y Seminario Jesús María. La metodología consistió en recolectar los residuos sólidos por espacio de cinco días en cada institución educativa y por cada nivel educativo (primaria y secundaria), el pesaje y segregación se realizó en el centro de acopio a partir de ello se determinó los siguientes parámetros la densidad, generación per cápita, composición física y humedad de los residuos sólidos. Además se aplicó una encuesta para identificar hábitos de consumo en los grupos humanos estudiados (docentes, estudiantes y personal administrativo) para determinar el número de muestras se utilizó la fórmula de muestreo estratificado por afijación proporcional ya que la densidad de los residuos está relacionada con los estilos de vida y costumbres de las personas .Los resultados del estudio de los residuos sólidos nos da a conocer la densidad de los residuos sólidos en la I.E San Juan de la Libertad es de $57,40 \mathrm{~kg} / \mathrm{m}^{3}$, Virgen Asunta $52,14 \mathrm{~kg} / \mathrm{m}^{3}$ y Seminario Jesús María con 54,24 kg/m³ , la generación per cápita en la I.E Seminario Jesús María es $0,05 \mathrm{~kg} / \mathrm{hab} \times$ día, en la I.E Virgen Asunta con $0,04 \mathrm{~kg} / \mathrm{hab} \times$ día y la I.E San Juan de la Libertad con $0,03 \mathrm{~kg} /$ hab $\times$ dia, el tipo de residuos sólidos que más generan son residuos orgánicos en las tres instituciones educativas, el promedio de la humedad de los residuos orgánicos en la I.E San Juan de la Libertad es 82,20\%, Virgen Asunta 78,72\% y Seminario Jesús María 75,06\%. los resultados de la encuesta nos dan a conocer que el 63,10\% de la I.E San Juan de la Libertad traen alimentos desde sus casas a la I.E., el tipo de alimentos que más traen son frutas con un 50,20\% y lo que más compran dentro de su I.E. son galletas con un 34.4\%, en la I.E. Virgen Asunta el 78,40\% traen alimentos desde sus casas a la I.E., el tipo de alimentos que más traen son frutas con $56,10 \%$ y lo que más compran en su I.E. es comida con un 49,60\%. Asimismo, en la I.E. Seminario Jesús María el 39,50\% traen alimentos desde su casa a la I.E, el tipo de alimentos que traen son frutas con un $39,50 \%$ y lo que más compran en la I.E. son comida con un $64,00 \%$.

Palabras claves: Residuos sólidos, instituciones educativas, generación per cápita, composición física y humedad.

\begin{abstract}
The objective of the present investigation was to determine the density of the solid waste generated in the educational institutions of San Juan de la Libertad, Virgen Asunta and Seminario Jesús María. The methodology consisted in collecting solid waste for five days in each educational institution and for each level of education (primary and secondary), the weighing and segregation was carried out in the collection center. From there, the following parameters were determined: density, generation per capita, physical composition and humidity of solid waste. In addition, a survey was applied to identify consumption habits in the human groups studied (teachers, students and administrative staff). To determine the number of samples, the sampling formula was used, stratified by proportional allocation, since the density of the waste is related to the lifestyles and customs of people. The results of the study of solid waste reveals the density of solid waste in the IE San Juan de la Libertad is $57.40 \mathrm{~kg} / \mathrm{m}^{3}$, Virgen Asunta 52.14 $\mathrm{kg} / \mathrm{m}^{3}$ and Seminar Jesús María with $54,24 \mathrm{~kg} / \mathrm{m}^{3}$, the generation per capita in the IE Jesús María Seminary is 0.05 $\mathrm{kg} / \mathrm{hab} \times$ day, in the IE Virgen Asunta with $0.04 \mathrm{~kg} / \mathrm{hab} \times$ day and I.E. San Juan de la Libertad with $0.03 \mathrm{~kg} / \mathrm{hab} \times$ day. , the type of solid waste that generates the most is organic waste in the three educational institutions, the average humidity of organic waste in the I.E. San Juan de la Libertad is $82.20 \%$, Virgen Asunta $78.72 \%$ and Jesús María Seminar $75.06 \%$. the results of the survey tell us that $63.10 \%$ of the IE San Juan de la Libertad bring food from their homes to the IE, the type of food they bring the most are fruits with $50.20 \%$ and what they buy the most within its IE they are cookies with $34.40 \%$, in the I.E. Virgen Asunta $78.40 \%$ bring food from their homes to the I.E., the type of food that they bring the most are fruits with $56.10 \%$ and what else they buy in their I.E. it's food with $49.60 \%$. Also, in the I.E. Seminario Jesús María 39.50\% bring food from their house to the I.E, the type of food they bring are fruits with $39.50 \%$ and what else they buy in the I.E. they are food with $64.00 \%$.
\end{abstract}

Keywords: Solid waste, educational institutions, generation per capita, physical composition and humidity.

\footnotetext{
${ }^{1}$ Universidad Nacional Toribio Rodríguez de Mendoza de Amazonas (UNTRM-A), Instituto de Investigación para el Desarrollo Sustentable de Ceja de Selva, Calle Higos Urco N³42-350-356, Calle Universitaria N³04, Chachapoyas, Perú

*Autor de correspondencia. E-mail: soliva@indes-ces.edu.pe
} 


\section{INTRODUCCIÓN}

La deficiente gestión de residuos sólidos impacta negativamente en el ambiente y en la salud de la población, generando la proliferación de insectos y roedores, así como también a malos olores derivados del proceso de descomposición el cual ejerce una influencia negativa directa en la salud. Es por ello que una gestión ecoeficiente municipal considera a los residuos sólidos como un recurso y su aprovechamiento como una actividad rentable ecológica y económicamente, que significa un cambio radical en el enfoque del manejo de residuos que pasa de recolectarlos para la disposición final a segregarlos para su utilización como materia prima en diferentes procesos productivos limpios incluido la generación de energía.

El primer paso que deben dar las instituciones educativas es conocer el tipo de problema con el que lidian y para ello es necesario que conozcan la densidad, cantidad y el tipo de residuos que están generando, pues la caracterización de los residuos es el primer paso que se debe dar para plantear un sistema de gestión integral de residuos sólidos (Armijo et al., 2006).

Los estudios referentes a la caracterización de los residuos sólidos en centros de educación son escasos.
El ECRS- Llata (2013), caracterizó los residuos sólidos en instituciones educativas en tres niveles y concluyó que la generación per cápita de los residuos sólidos en instituciones educativas nivel inicial es de 0,1 Kg/alumno/día; nivel Primaria de 0,02 $\mathrm{Kg}$ /alumno/día y secundaria de $0,4 \mathrm{Kg}$ /alumno/día. Así mismo determinó que el componente físico que existe en mayor cantidad dentro de los residuos sólidos domiciliarios del distrito de Llata es la materia orgánica alcanzando el 29,6\%, así mismo un 13,4\% residuos de madera y follaje, seguido de residuos inertes con un $9,5 \%$, el $8,7 \%$ de residuos tipo vidrio, un $8,6 \%$ de residuos sanitarios, un $7,7 \%$ de residuos tipo metal, un $6,4 \%$ de residuos tipo bolsas

Pacheco et al., (2011) En su trabajo de investigación “Caracterización del problema de la Gestión de Residuos Sólidos en la UNMSM" concluyó que la densidad de los residuos generados en la UNMSM es de 400 $\mathrm{kg} / \mathrm{m}^{3}$, con una producción per cápita de 0,65 $\mathrm{kg} / \mathrm{hab} /$ día, el residuo de mayor generación serían los restos de comida (30\%), seguido de papel, cartones y similares $(18,1 \%)$.

La presente investigación aporta información sobre la densidad de los residuos sólidos generados en las instituciones educativas San Juan de la libertad, Virgen Asunta y Seminario Jesús de María de la ciudad de

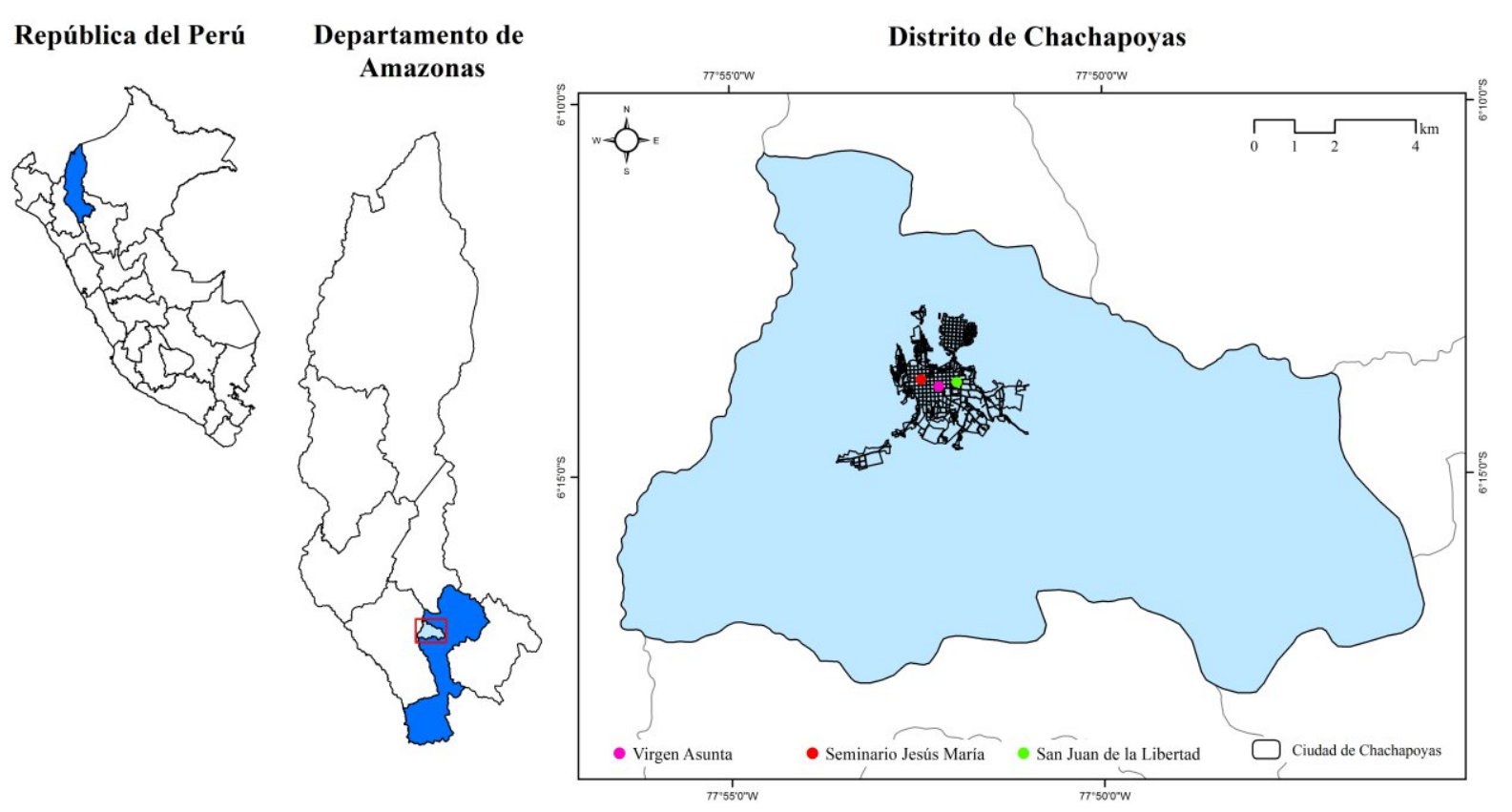

Figura 1. Ubicación de las instituciones educativas en la ciudad de Chachapoyas, provincia de Chachapoyas, Departamento Amazonas. 
Chachapoyas, la misma que es un medio fundamental e importante para generar datos sobre la densidad y cantidad de residuos sólidos generados en cada institución educativa, datos que servirán para elaborar un plan de manejo de residuos sólidos en las instituciones educativas.

Durante el desarrollo de este trabajo se cumplió con el objetivo general determinar la densidad de los residuos sólidos generados en las instituciones educativas, San Juan de la Libertad, Virgen de Asunta y Seminario Jesús María de la ciudad de Chachapoyas.

Asimismo, se tiene la hipótesis en que la densidad de residuos sólidos generados en la institución educativa San Juan de la Libertad es mayor que la densidad de los residuos sólidos generados en las instituciones educativas Virgen de Asunta y Seminario Jesús María.

\section{MATERIALES Y MÉTODOS}

\section{Ubicación del área de estudio}

La presente investigación se desarrolló en las Instituciones educativas San Juan de la Libertad, Virgen Asunta y Seminario Jesús María de la ciudad de Chachapoya, Provincia de Chachapoyas departamento de Amazonas. (Figura 1).

\section{Metodología empleada}

La población para el estudio fueron todos los residuos sólidos generados por los estudiantes, docentes y personal administrativo de ambos niveles educativos, se recolectaron todos los residuos sólidos generados en el nivel primario y secundario durante cinco días consecutivos (lunes-viernes). A las 6.00 am se procedió a colocar las bolsas debidamente codificadas en los contenedores en las diferentes áreas de las instituciones educativas, luego se recogió a las 12:30 pm correspondiente al nivel secundaria, para inmediatamente colocar una bolsa nueva para ser recogida a las 6.30 pm de la tarde del nivel primaria. Los residuos sólidos generados en el turno noche fueron recogidos antes de colocar la primera bolsa para no alterar la muestra. Se procedió a realizar el pesado de cada una de las bolsas de residuos sólidos, todos estos datos fueron registrados en el cuaderno de campo, previa identificación de

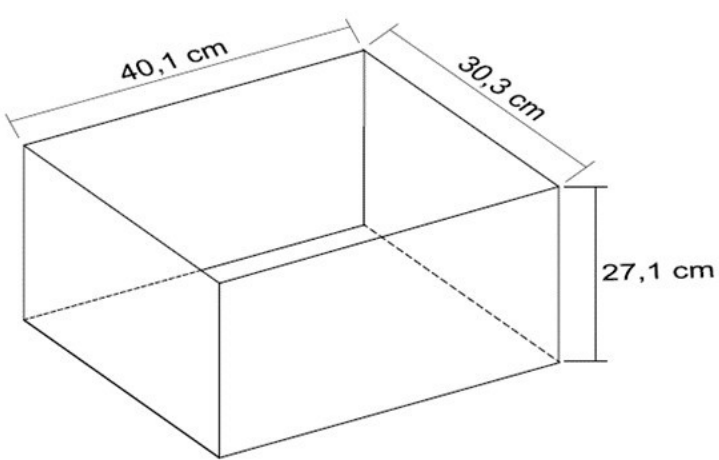

Figura 2. Dimensionamiento de la caja de vidrio.

los códigos. Para determinar la densidad de los residuos sólidos se utilizó una caja de vidrio (Figura 2).

Se colocó los residuos sólidos en la caja de vidrio de volumen conocido, uniformizando la muestra, este procedimiento se realizó durante 5 días consecutivos y por nivel educativo. El cálculo de la densidad de los residuos, empleando la siguiente fórmula (MINAM, 2008).

$$
\text { Densidad suelta }\left(\frac{\mathrm{kg}}{\mathrm{m}^{3}}\right)=\frac{W}{V}
$$

Donde:

$\mathrm{W}=$ Peso de los residuos solidos

$\mathrm{V}=$ Volumen del residuo sólido

\section{Determinación de la generación per cápita}

Para determinar la generación per cápita (GPC) se pesó todas las bolsas de los residuos de cada institución educativa y se procedió a determinar la generación per cápita aplicando la siguiente fórmula (Jarami1lo, 2002).

$G P C=\frac{\text { día } 1+\text { día } 2+\text { día } 3+\text { día } 4+\text { día } 5}{\text { Número de personas } \times 5 \text { dias }}=\frac{\mathrm{Kg}}{\text { persona } / \text { día }}$

\section{Determinación de la Composición Física}

Se procedió a vaciar el contenido de la caja de vidrio para clasificar los materiales por tipo, los componentes clasificados se depositaron en bolsas, para ser pesados, una vez obtenidos los datos se realizó la disposición final. 


\section{Determinación de la humedad.}

Para determinar la humedad se utilizó el residuo orgánico segregado anteriormente realizando el método del cuarteo, hasta obtener una muestra de 250 gr, este mismo procedimiento se repitió durante 5 días consecutivos por cada nivel.

La muestra recolectada, previamente pesada (250 gr), se picó en pequeños trozos hasta conseguir una muestra uniforme y luego se envasó en una bolsa hermética para ser llevado a los Laboratorio del Instituto de Investigación para el Desarrollo Sustentable de Ceja de Selva (INDES-CES) de la Universidad Nacional Toribio Rodríguez de Mendoza (UNTRM). Las muestras se vaciaron en cajas de cartón debidamente pesadas y rotuladas para ser secadas en la estufa a una temperatura de $80^{\circ} \mathrm{C}$. Una vez las muestras bien secas se realizaron el pesaje para luego realizar el cálculo correspondiente (Figura 3)

Para determinar la humedad se aplicó la siguiente

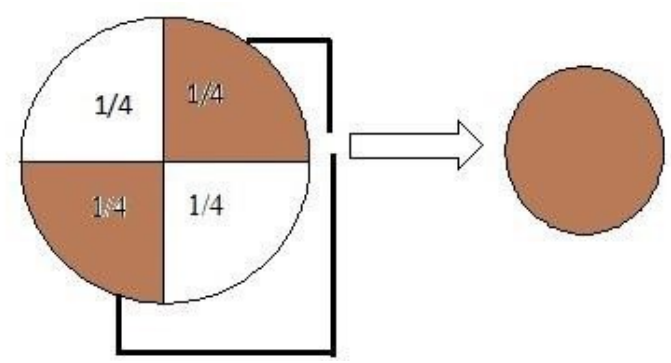

Figura 3. Método del cuarteo (250 g de $1 \mathrm{~kg})$.

fórmula (Kunitoshi-Sakurai, 1983).

$\%$ Humedad de los resiudos sólidos $=\frac{w 2-w 3}{w 2-w 1} \times 100$

Donde:
$\mathrm{W} 2=$ Peso húmedo

$\mathrm{W} 3=$ Peso seco

$\mathrm{W} 1=$ Peso de la caja

\section{Encuesta de hábitos de consumo y residuos sólidos} Se determinó un número de muestras de estudiantes, docentes y personal administrativo, para ser encuestados para determinar el número de muestras se aplicó la siguiente formula muestreo estratificado por afijación proporcional, (Cochran, 1998).

$$
n h=\frac{1}{\left(N \times \frac{e}{z_{1-\alpha}}\right)^{2}} * \sum N h \times p h \times q h
$$

Donde:

$\mathrm{n}=$ Número de muestras $=$ ?

$\mathrm{N}=$ Población total $=3820$

$\mathrm{e}=$ Error permisible $=0,07$

$\mathrm{z}=$ Valor de la distribución normal asociado al nivel de confianza $=1,96$

$\mathrm{p}=$ Probabilidad que evidencia menores hábitos de consumo $=0,49$

$\mathrm{q}=$ Probabilidad que evidencia menores hábitos de consumo $=0,51$

$\mathrm{h}=$ Estratos $=3$

$(1-\alpha)=$ Nivel de confianza $=0,95$

\section{Aplicación de la encuesta}

En la tabla 3 nos muestra la cantidad de docentes, estudiantes y administrativos que fueron encuestados en cada institución educativa. Estos datos se obtuvieron a partir de la fórmula de muestreo estratificado por afijación proporcional, (Cochran, 1998).

Tabla 1. Número de encuestas aplicadas en cada institución educativa

\begin{tabular}{cccc}
\hline & San Juan de la Libertad & Virgen Asunta & Seminario Jesús María \\
\hline Docentes & 11 & 6 & 6 \\
Estudiantes & 226 & 132 & 107 \\
Administrativos & 4 & 1 & 1 \\
\hline Total & 241 & 139 & 114 \\
\hline
\end{tabular}




\section{Composición física de los residuos sólidos}

En las figuras 4 a 7 se observa que en las tres instituciones educativas el tipo de residuo sólido que más generan son residuos orgánicos.Tipo de residuos sólidos que más se genera en cada institución

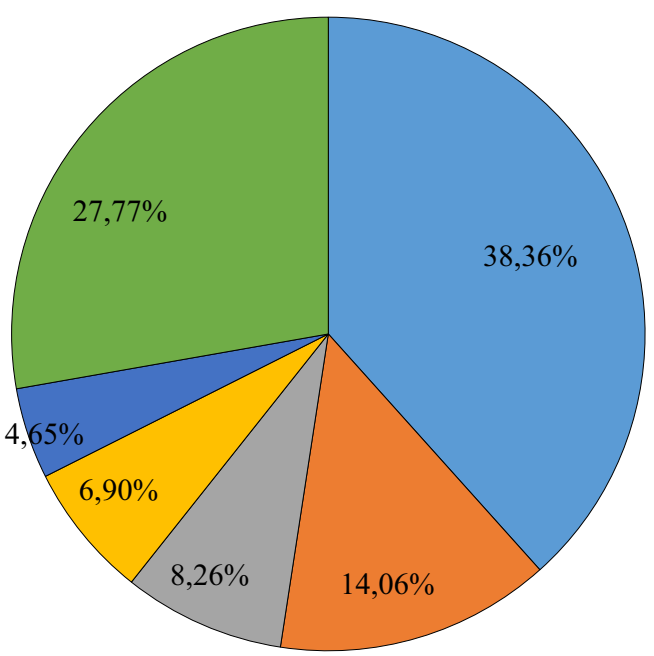

$\begin{array}{lc}\text { Residuos Orgánicos } & \text { Papel blanco } \\ \text { Latas } & \text { PET } \\ \text { Cartón mixto } & \text { Otros }\end{array}$

Figura 4. Composición física de los residuos sólidos I.E. San Juan de la Libertad.
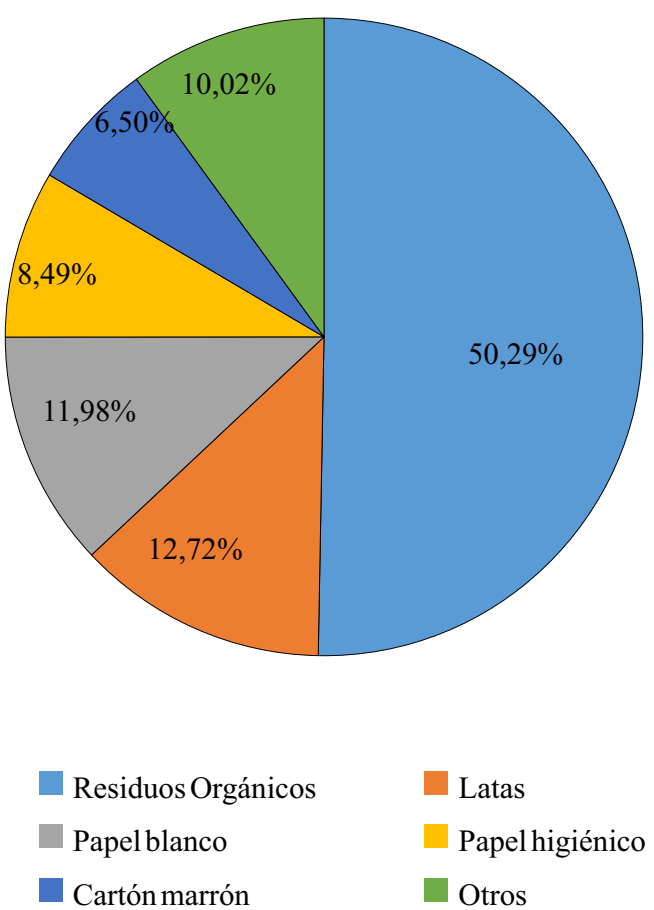

Figura 5. Composición física de los residuos sólidos I.E Virgen Asunta.

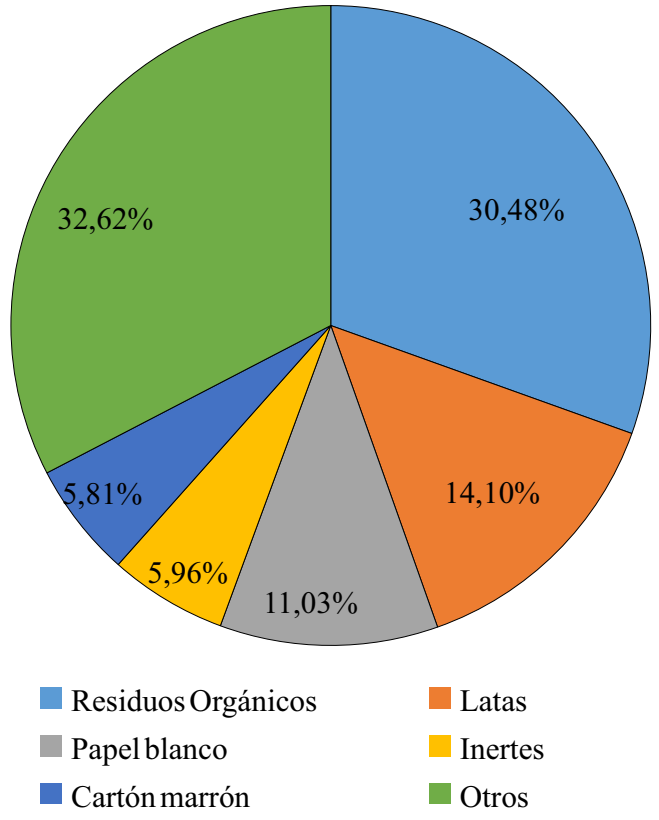

Figura 6. Composición física de los residuos sólidos I.E Seminario Jesús María

El la figura 7 , nos da a conocer sobre el tipo de residuos sólidos que más generan en las tres instituciones educativas: Teniendo como resultado que el tipo de residuos sólidos que más se generan son papeles, siendo el mayor porcentaje en la I.E Virgen Asunta con un $65,50 \%$, San Juan de la Libertad con un 59,80\% y Seminario Jesús María con 57,00\%. Por otro lado, el promedio de humedad más alto se encontró en San Juan de la Libertad con un 82,20\%, seguido por Virgen Asunta con 78,72\% y Seminario Jesús María con un $75,06 \%$. Por otro lado la generación per cápita (GPC) más alta, se encontró en la institución Seminario Jesús María con un $0,05 \%$, seguido con un $0,04 \%$ y un $0,03 \%$ para las instituciones de Virgen Asunta y San Juan de la Libertad respectivamente.

Así mismo se tiene como resultado, el menor porcentaje de residuos sólidos generados en las tres instituciones educativas son vasos descartables con $1,8 \%$ en la I.E San Juan de la Libertad, 1,4\% Virgen Asunta y $0,9 \%$ en el I.E Seminario Jesús María $0,9 \%$. 


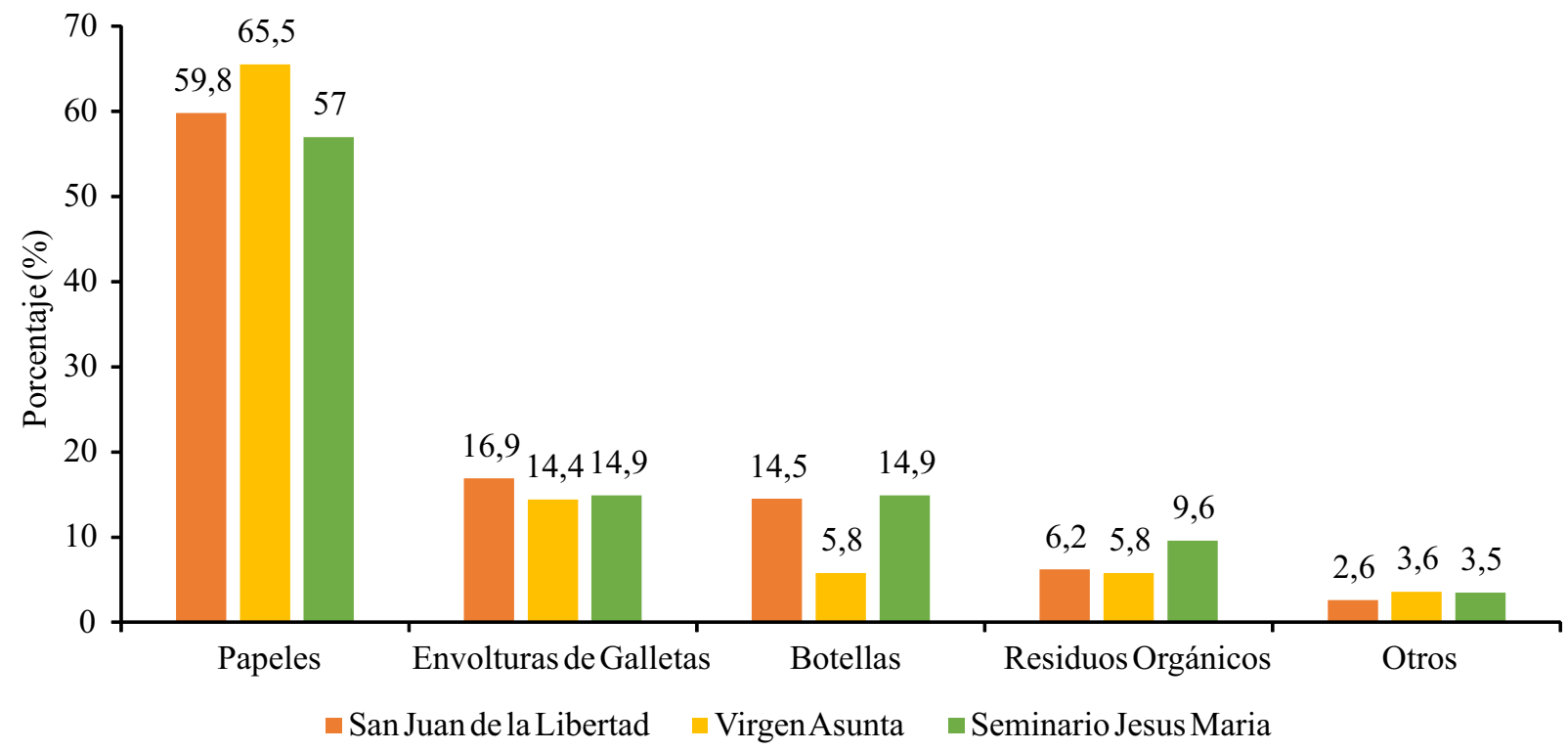

Figura 7. Residuos sólidos más generados en cada institución educativa

\section{IV.DISCUSIÓN}

Las densidades de residuos sólidos generados en la I.E. San Juan de la Libertad es de $57,40 \mathrm{~kg} / \mathrm{m} 3$, I.E. Virgen Asunta 52,14 kg/m3 y la I.E. Seminario Jesús María 54,24 kg/m3. Estos valores son menores a los reportados por Gonzales et al. (2010), en la caracterización de los residuos sólidos en la ciudad universitaria de Paturpampa de la Universidad Nacional de Huancavelica encontrando una densidad de $391,24 \mathrm{~kg} / \mathrm{m}^{3}$. Esto es corroborado por (CEPIS, 1997) donde nos dice que la densidad sin compactar puede variar hasta en un $50,00 \%$ respecto a los valores representativos, de acuerdo a la naturaleza de los componentes y su contenido de humedad.

Además, los datos reportados por la Municipalidad distrital de Breña (2016) en el estudio de caracterización de residuos sólidos municipales se encontró que la densidad promedio ( $\sin$ compactar) de los residuos sólidos no domiciliarios es $215,66 \mathrm{~kg} / \mathrm{m}^{3}$, siendo también valores mayores a los que se obtuvo en esta investigación.

Similar a lo reportado en la presente investigación la Municipalidad provincial de Chumbivilcas (2015), encontró que la densidad de residuos sólidos no domiciliarias sin compactar del Distrito de Santo Tomas es de $72,62 \mathrm{~kg} / \mathrm{m}^{3}$.

La municipalidad provincial de Trujillo (2016) en su investigación estudio de caracterización de residuos sólidos municipales del área urbana del distrito Trujillo la menciona que la densidad no compactada de los residuos sólidos no domiciliarios del distrito de Trujillo es de $292,11 \mathrm{~kg} / \mathrm{m}^{3}$.

La generación per cápita de residuos sólidos en las tres instituciones tienen valores mayores a los reportados por la municipalidad provincial de Puno (2013), en su investigación estudio de caracterización de residuos sólidos del distrito de Puno, encontrando una producción per cápita de $0,11 \mathrm{~kg} /$ alumno/dia en el nivel primaria y secundaria.

Carrillo (2007), en su investigación caracterización de los residuos sólidos de la Universidad de Michoacán (México) encontró una producción per cápita de 0,01 kg/día y Gonzales et al. (2010), en su investigación estudio de caracterización de los residuos sólidos en la ciudad universitaria de Paturpampa de la Universidad Nacional de Huancavelica" encontró una producción per cápita es de $0,16 \mathrm{~kg} /$ persona/día, siendo estos valores menores a la generación per cápita encontrada en esta investigación.

Sin embargo, la producción per cápita encontrada en este estudio son menores a los encontrados por Pacheco et al., (2011) en la caracterización del problema de la gestión de residuos sólidos en la UNMSM", obteniendo como resultado una producción per cápita de 
$0,65 \mathrm{~kg} / \mathrm{hab} \times$ dia. y la Municipalidad distrital de Ate (2011), en su investigación estudio de Caracterización de residuos sólidos no domiciliarios del distrito de Ate obtuvo una generación de residuos sólidos por alumno de 0,09 kg/alumno/día.

Eco consultorías e ingeniería SAC. (2013), realizó una investigación estudio de caracterización física de residuos sólidos municipales en la ciudad de Piura, encontrando que en el nivel primaria la generación per cápita es de 0,02 kg/alumno/día y en el nivel secundario $0,01 \mathrm{~kg} /$ alumno/día, siendo estos valores menores a los datos encontrados en esta investigación.

En las tres instituciones educativas hay un mayor porcentaje de residuos orgánicos. El porcentaje de residuos sólidos orgánicos en la I.E. Virgen de Asunta es de 50,29\%, San Juan de la Libertad 38,36\% y Seminario Jesús María con un 30,48\%, los valores obtenidos por esta investigación son corroborados por Jaramillo (2002), quien indica que los residuos orgánicos putrescibles se encuentran entre un 30 a un $60 \%$ del total de residuos generados en domicilios en zonas urbanas, mientras que en zonas semi rurales y rurales este porcentaje se incrementa a un 50 a $80 \%$ debido a los hábitos de consumo de las familias. Los residuos orgánicos sirven como materia prima para elaborar compost, que se puede emplear para incrementar la producción de los cultivos propios o comercializarlos, generando ingresos para la sostenibilidad de esta actividad de reciclaje.

Así mismo el papel blanco es generado en mayor porcentaje en la I.E. San Juan de la Libertad con un 14,06\%, I.E. Virgen Asunta con un 11,98\% y en la I.E. Seminario Jesús María con un 11,03\%. este valor es corroborado por el trabajo de Barrena et al (2013), donde menciona que el tipo de residuo solidos que mayor generan en el Campus de la UNTRM son papeles con un $12,61 \%$ del total de residuos sólidos producidos.

El porcentaje de la humedad de los residuos orgánicos de la I.E San Juan de la Libertad es 82,2\%, I.E Virgen Asunta es $78,72 \%$ y la I.E seminario Jesús María es $75,06 \%$, siendo estos valores mayores a la humedad de residuos sólidos encontrados por Eco consultorías e ingeniería SAC. (2013), en su investigación estudio de caracterización física de residuos sólidos municipales en la ciudad de Piura, donde la humedad es de 50,11\%.

\section{CONCLUSIONES}

Los residuos sólidos generados en cada institución por los entrevistados en la institución educativa San Juan de la Libertad el $34,40 \%$, lo que más compran dentro de su I.E son galletas, en la I.E Virgen Asunta el $49,60 \%$ compran comida y en el Seminario Jesús María el 64,00\% compran comida, con respecto a la frecuencia con la que consumen gaseosas, en las tres I.E consumen dos veces a la semana, I.E San Juan de la Libertad el 44,40\%, virgen asunta 38,80\% y Seminario Jesús María 36,00\%. Así mismo el tipo de alimento que más traen son frutas con un porcentaje de 56,10\% en la I.E Virgen Asunta, 50,20\% en la I.E San Juan de la Libertad y el 39,50\% I.E Seminario Jesús María. Estos datos, permite determinar cuáles son los residuos que más impactan en el flojo de residuos sólidos para elaborar planes de acción más certeros. Además, los tipos de residuos que se generan en cada institución pueden ser reciclados o rehusados en su gran mayoría.

\section{RECOMENDACIONES}

Realizar talleres de educación ambiental permanentes a los estudiantes, docentes, personal administrativos y padres de familia sobre el reaprovechamiento y manejo adecuado de los residuos sólidos.

Realizar talleres educativos a los padres de familia sobre loncheras saludables, logrando que se interesen por la nutrición de sus hijos y así mejorar hábitos alimenticios, ya que la alimentación saludable y adecuada es imprescindible en el desarrollo de un estudiante. Implementar contenedores de colores según la norma técnica para clasificar y según el tipo de residuos sólidos encontrados en esta investigación.

Sistematización del estudio de los residuos sólidos cada dos años para evaluar el comportamiento del manejo de los residuos sólidos.

Realizar compostaje con los residuos orgánicos gene- 
rados y poder aprovecharlos en las áreas verdes.

Reciclar los residuos sólidos para comercializarlos y así obtener ingresos económicos para implementar contenedores de almacenamiento de residuos sólidos.

Realizar manualidades de los diferentes tipos de residuos sólidos que se generan en las instituciones educativas.

\section{REFERENCIAS BIBLIOGRÁFICAS}

Armijo, C., S. Ojeda-Benítez, E. Ramírez-Barreto y A. Quintanilla-Montoya. 2006. "Potencial de reciclaje de los residuos de una institución de educación superior: el caso de la Universidad Autónoma de Baja California.” Ingeniería, RevistaAcadémica 10 (3): 13-21.

Barrena, M. A. 2013. Caracterización y Segregación de residuos sólidos del campus de la Universidad Nacional Toribio Rodríguez de Mendoza de Amazonas. Informe Técnico. Universidad Nacional Toribio Rodríguez de Mendoza. Chachapoyas (Perú)

Cochran, W. 1998. Técnicas de Muestreo. Ciudad de México (México): Col. Asturias

ECO Consultorías e Ingeniería SAC. 2013. Estudio de caracterización física de residuos sólidos municipales de la ciudad de Piura. Informe Técnico. Piura (Perú)

ECRS-Llata. 2013. Estudio de Caracterización de Residuos Sólidos Municipales del distrito de Llata, provincia de Huamaliés, departamento de Huánuco. Informe Técnico. Llata (Perú).

Gonzales T. y V. Sánchez 2010. Caracterización de los Residuos Sólidos en la Ciudad Universitaria de Paturpampa de la Universidad Nacional de Huancavelica. Informe Técnico Huancavelica (Perú).

Jaramillo, J. (2002). Una solución para la disposición final de residuos sólidos municipales en pequeñas poblaciones. Antioquia (Colombia): CEPIS/OPS/OMS.

MINAM. 2008. Guía metodológica para la elaboración del estudio de caracterización de residuos sólidos municipales (EC-RSM). Informe Técnico. Lima (Perú).

Municipalidad distrital de Ate. 2011. Estudio de caracterización de residuos sólidos domiciliarios del distrito de Ate. Informe Técnico. Lima (Perú).

Municipalidad distrital de Breña. 2016. Estudio de caracterización de residuos sólidos municipales/Gerencia de servicios comunales y gestión ambiental. Informe Técnico. Lima (Perú).

Municipalidad provincial de Chumbivilcas. 2015. Estudio de caracterización de residuos sólidos del distrito de Santo Tomás de la provincia de Chumbivilcas. Informe Técnico. Cusco (Perú).

Municipalidad provincial de Puno. 2013. Estudio de caracterización de residuos sólidos del distrito de Puno. Informe Técnico. Puno (Perú)

Municipalidad distrital de Rimac. 2013. Plan de manejo de residuos sólidos. Sub gerencia de limpieza pública y control ambiental. Informe Técnico. Lima (Perú)

Municipalidad provincial de Trujillo 2016. Estudio de caracterización de residuos sólidos municipales del área urbana del distrito de Trujillo. Informe Técnico. Trujillo (Perú)

Pacheco A., J. Espinoza, W. Arévalo y S. Iglesias. 2011. “Caracterización del problema de la Gestión de Residuos Sólidos en la UNMSM." Revista del Instituto de Investigación de la Facultad de Ingeniería Geológica, Minera, Metalurgica y Geográfica 14(27).

Sakurai, K. 1983. Manual de instrucción de análisis de residuos sólidos municipales. Lima (Perú): CEPIS. 